\title{
Uso racional de antimicrobianos e impacto no perfil de resistência microbiológica em
} tempos de pandemia pela Covid-19

\author{
Rational use of antimicrobials and impact on the microbiological resistance profile in times of \\ pandemic by Covid-19 \\ Uso racional de antimicrobianos e impacto en el perfil de resistencia microbiológica en tiempos de \\ pandemia por Covid-19
}

\author{
Rafael Ferreira Mesquita \\ ORCID: https://orcid.org/0000-0002-6914-0410 \\ Hospital São Camilo Fortaleza, Brasil \\ Hospital São José, Brasil \\ E-mail: Rafael.f.mesquita@terra.com.br \\ Cicero Allan Landim de Oliveira Lima \\ ORCID: https://orcid.org/0000-0001-6109-6382 \\ Hospital São José, Brasil \\ E-mail: allanlandim@gmail.com \\ Luan Victor de Almeida Lima \\ ORCID: https://orcid.org/0000-0002-2145-009X \\ Hospital São José, Brasil \\ Centro Universitário Christus, Brasil \\ E-mail: luanvictor.al@gmail.com \\ Bruno Pinheiro Aquino \\ ORCID: https://orcid.org/0000-0001-7434-3637 \\ Hospital São José, Brasil \\ E-mail: brunopinheiroa@gmail.com \\ Melissa Soares Medeiros \\ ORCID: https://orcid.org/0000-0002-5881-1485 \\ Hospital São Camilo Fortaleza, Brasil \\ Hospital São José, Brasil \\ Centro Universitário Christus, Brasil \\ E-mail: melmedeiros@hotmail.com
}

\begin{abstract}
Resumo
O uso racional de antimicrobianos tem sido uma das principais medidas para evitar o aumento de resistência antimicrobiana no mundo. O objetivo do presente estudo foi avaliar a utilização de antimicrobianos em um hospital privado de Fortaleza/Brasil e correlacionar com a crescente resistência antimicrobiana. Foram determinados dados quantitativos sobre infecções bacterianas, perfil microbiológico e uso de antimicrobianos obtidos de pacientes atendidos nos setores, entre janeiro de 2016 e agosto de 2021. Foram emitidos 2.733 pareceres para avaliação de uso de antibióticos em 2020 e em 2021 foram 2.044. Durante o ano de 2020 a utilização de antimicrobianos foi mais frequente entre maio e junho, e em 2021 nos meses de março e abril, sendo estes referidos meses os que refletem os pacientes com internação prolongada pós-Covid-19 e múltiplas infecções bacterianas secundárias. Observa-se também nesse período maior prescrição de Polimixina B e glicopeptídeos. Ao avaliar o perfil de sensibilidade dos Gram negativos, para P. aeruginosa observa-se uma redução drástica no último ano da sensibilidade a Piperacilina/tazobactam e Carbapenens, bem como a Polimixina B (91,7\% de sensibilidade). Para K. pneumoniae os resultados foram ainda piores, evidenciando alta resistência aos Carbapenens e Polimixina B, cuja sensibilidade do último foi de apenas 76,2\%, dificultando a escolha de opções terapêuticas. Detectou-se redução da sensibilidade a Cefatzidima/avibactam para P. aeruginosa, K. pneumoniae e Serratia sp. Concluiu-se que o aumento na prescrição de antimicrobianos de amplo espectro durante a pandemia por Covid-19 podem ter impactado no aumento da resistência bacteriana principalmente para Gram negativos. Palavras-chave: Antimicrobianos; Resistência; Covid-19; Pseudomonas aeruginosa; Klebsiella pneumoniae; Carbapenem.
\end{abstract}

\begin{abstract}
The rational use of antimicrobials has been one of the main measures to prevent the increase in antimicrobial resistance in the world. The aim of the present study was to evaluate the use of antimicrobials in a private hospital in Fortaleza/Brazil and to correlate with the increasing antimicrobial resistance. Quantitative data on bacterial infections,
\end{abstract}


microbiological profile and use of antimicrobials were determined, obtained from patients treated in the sectors, between January 2016 and August 2021. 2,733 reports were issued to evaluate the use of antibiotics in 2020 and in 2021 there were 2,044. During 2020, the use of antimicrobials was more frequent between May and June, and in 2021 in March and April, these months reflecting patients with prolonged hospitalization after Covid-19 and multiple secondary bacterial infections. During this period, there is also a greater prescription of Polymyxin B and glycopeptides. When evaluating the Gram-negative sensitivity profile for P. aeruginosa, a drastic reduction in the last year of sensitivity to Piperacillin/tazobactam and Carbapenens, as well as to Polymyxin B (91.7\% sensitivity) is observed. For K. pneumoniae, the results were even worse, showing high resistance to Carbapenens and Polymyxin B, whose sensitivity of the latter was only $76.2 \%$, making the choice of therapeutic options difficult. A reduction in sensitivity to Cefatzidime/avibactam was detected for P. aeruginosa, K. pneumoniae and Serratia sp. It was concluded that the increase in the prescription of broad-spectrum antimicrobials during the Covid-19 pandemic may have had an impact on the increase in bacterial resistance, mainly for Gram negatives.

Keywords: Antimicrobials; Resistance; Covid-19; Pseudomonas aeruginosa; Klebsiella pneumoniae; Carbapenem.

\section{Resumen}

El uso racional de antimicrobianos ha sido una de las principales medidas para prevenir el aumento de la resistencia a los antimicrobianos en el mundo. El objetivo del presente estudio fue evaluar el uso de antimicrobianos en un hospital privado en Fortaleza / Brasil y correlacionarlo con el aumento de la resistencia a los antimicrobianos. Se determinaron datos cuantitativos sobre infecciones bacterianas, perfil microbiológico y uso de antimicrobianos, obtenidos de pacientes atendidos en los sectores, entre enero de 2016 y agosto de 2021 . Se emitieron 2.733 opiniones para evaluar el uso de antibióticos en 2020 y en 2021 fueron 2.044. Durante 2020, el uso de antimicrobianos fue más frecuente entre mayo y junio, y en 2021 en marzo y abril, estos meses reflejan pacientes con hospitalización prolongada por Covid-19 y múltiples infecciones bacterianas secundarias. Durante este período, también hay una mayor prescripción de polimixina B y glicopéptidos. Al evaluar el perfil de sensibilidad gramnegativa para P. aeruginosa, se observa una reducción drástica en el último año de la sensibilidad a Piperacilina / tazobactam y Carbapenens, así como a Polimixina B (91,7\% de sensibilidad). En el caso de K. pneumoniae, los resultados fueron aún peores, mostrando una alta resistencia a los carbapenos y la polimixina B, cuya sensibilidad de esta última era de solo el 76,2\%, lo que dificultaba la elección de las opciones terapéuticas. Se detectó una reducción en la sensibilidad a Cefatzidime / avibactam para P. aeruginosa, K. pneumoniae y Serratia sp. Se concluyó que el aumento en la prescripción de antimicrobianos de amplio espectro durante la pandemia de Covid-19 puede haber tenido un impacto en el aumento de la resistencia bacteriana, principalmente para Gram negativos.

Palabras clave: Antimicrobianos; Resistencia; Covid-19; Pseudomonas aeruginosa; Klebsiella pneumoniae; Carbapenem.

\section{Introdução}

O uso racional de antimicrobianos pode ser definido como a prática de prescrição que resulta na ótima indicação, dosagem, via de administração e duração de um esquema terapêutico ou profilático, propiciando o alcance de sucesso clínico com mínima toxicidade para o paciente e reduzido impacto sobre a resistência microbiana (Dandolini et al., 2013; Mota et al., 2008). Portanto, atualmente é de extrema importância valorizar as políticas institucionais que visam o melhor controle da utilização de antimicrobianos durante a internação hospitalar de um paciente (Aquino, 2008). Pois, o desfecho negativo mais preocupante seria o aumento de mortalidade (Pinto et al., 2012). Um relatório recente de grande visibilidade, publicado em 2014 por Lord Jim O’Neill e seu grupo, estima que, em 2050, 10 milhões de pessoas morrerão todos os anos devido à resistência antimicrobiana (RA), a menos que uma resposta global ao problema seja instituída (de Kraker et al., 2016; O'Neill, 2014). Sem dúvida, há uma grande carga clínica e de saúde pública associada à RA, mas é um desafio quantificar o excesso de morbimortalidade associado. Em geral, utilizamos para determinar a carga da RA através da estimativa do número de infecções causadas por patógenos que são resistentes ao antibiótico (ou classe de antibióticos) de interesse, a fim de traçar o melhor entendimento sobre o nosocômio em estudo. Claramente, há uma necessidade de estimativas de carga RA mais confiáveis, incluindo limites incertos e modelagem mais cuidadosa de cenários futuros, incluindo análises de sensibilidade. O principal prérequisito para isso são dados de vigilância de RA mais abrangentes, especialmente para países de baixa e média renda, e especialmente para infecções adquiridas na comunidade (de Kraker et al., 2016).

A utilização indiscriminada de antimicrobianos pela comunidade tem sido um dos maiores desafios para o uso racional e controle da RA, e provavelmente uma boa parte dessa responsabilidade possa recair sobre a dificuldade de entendimento e 
seguimento das prescrições médicas pelos usuários (Rauber et al., 2009). Em um estudo, detectou-se uma grande dificuldade de entendimento da prescrição, especialmente por usuários sem estudo formal, chegando a níveis de entendimento de apenas 21 a 28\% das prescrições estabelecidas (Fiol et al., 2010). Portanto, a pressão seletiva sobre os patógenos se inicia a nível comunitário, levando a chegada ao nosocômio de pacientes previamente portando bactérias com RA. O que diferencia um antimicrobiano de qualquer outro medicamento terapêutico é a sua capacidade de perder eficácia ao longo do tempo, resistência por pressão seletiva, sendo necessária a substituição constante destes por meio da descoberta de novos agentes (Luepke et al., 2013). Segundo a Organização Mundial de Saúde, a resistência aos antibióticos é extremamente disseminada em todo o mundo, tanto em países de alta como de baixa renda. Escherichia coli, Staphylococcus aureus e Streptococcus pneumoniae são as bactérias resistentes mais comumente relatadas (WHO, 2018).

Vários são os fatores que têm sido apontados como causadores da resistência bacteriana, tais como: a globalização, como facilitador da transmissão dos mecanismos de resistência aos patógenos em todo o mundo; o uso abusivo ou inadequado de antibióticos; o uso massivo de antimicrobianos em animais agrícolas; e a prescrição arbitrária desses medicamentos (Hawkey, et al., 2015; Helke, et al., 2016). É importante observar que o aumento da RA tem impacto direto na morbimortalidade dos pacientes, devido à redução das opções terapêuticas e aos períodos prolongados de internação e alto custo, com consequências determinantes para o sistema de saúde (Kalpoe, et al., 2012). Durante a internação hospitalar, os principais fatores de risco para o paciente portar RA são: idade, hospitalização anterior, presença de cateter e outros devices invasivos, residência em casa de saúde, infecções anteriores, exposição a antibióticos ou algumas comorbidades, como diabetes ou imunossupressão (Opatowski et al., 2021). A importância dos "dados do mundo real", derivados de registros manuais e eletrônicos de saúde ou bancos de dados administrativos, que complementam os dados de ensaios clínicos convencionais no processo de tomada de decisão regulatória, tem sido extremamente valorizado (FDA, CDRH, CBER, 2017).

Durante a pandemia por Covid-19 a utilização em larga escala de antimicrobianos pode ter sido responsável pelo aumento de RA. Covid-19 e RA podem impactar um ao outro, pois há um impulso para recorrer aos antimicrobianos existentes em pacientes com Covid-19 gravemente enfermos na ausência de tratamentos específicos, enquanto as tentativas de controlar a disseminação de Covid-19 também podem levar a uma redução da resistência antimicrobiana (Nieuwlaat et al., 2021). Embora o impacto do Covid-19 na RA ainda não seja totalmente compreendido, é importante ter uma visão geral das primeiras descobertas sobre o Covid-19 e a RA, incluindo o uso de antibióticos, e fornecer recomendações e lições aprendidas para melhorar o manejo antimicrobiano (Adebisi et al., 2021).

Diante da importância e do impacto do uso de antimicrobianos na flora microbiológica de uma instituição, o objetivo do presente estudo foi avaliar a utilização de antimicrobianos em um hospital privado de Fortaleza/CE/Brasil e correlacionar com a crescente resistência antimicrobiana a nível nosocomial. Com o impacto da pandemia por Covid-19 desde 2020, busca-se detectar possíveis alterações de RA em patógenos que comumente levam a infecção relacionada a serviços de saúde.

\section{Metodologia}

\subsection{Tipo de estudo}

O estudo é uma pesquisa exploratória, realizada por meio de uma metodologia transversal e observacional. Na saúde, seja na saúde pública ou na medicina, os levantamentos mais frequentes são feitos na forma de estudos observacionais, pois os pesquisadores não interferem nos fenômenos em estudo, apenas os observam de forma sistemática e padronizada, coletando e registrando informações, dados ou materiais (como os resultados e identificação de patógenos multirresistentes) que ocorrem espontaneamente em um dado momento do processo saúde-doença, ou durante sua evolução natural, para então proceder à sua descrição e / ou análise (Zangirolami-Raimundo et al., 2018; Song, \& Chung. 2010). 
Os dados quantitativos sobre infecções bacterianas, perfil microbiológico e uso de antimicrobianos foram obtidos de pacientes atendidos nos setores: emergência, unidade de terapia intensiva adulta e neonatal e clínica médica, do Hospital São Camilo de Fortaleza/CE, entre janeiro de 2016 e agosto de 2021. A identificação bacteriana e a avaliação da suscetibilidade aos antimicrobianos foram realizadas pelo sistema automatizado Phoenix e microdiluição em caldo para a Polimixina. Foram avaliados dados sobre o consumo de antibióticos de controle pelo Serviço de Controle de Infecção Hospitalar (SCIH) de 2020 a 2021. As tabelas e gráficos foram desenhadas a partir de uma planilha de Excel. A Análise do perfil microbiano por patógeno foi realizada entre os anos de 2016 e 2021.

\subsection{Local e período de estudo}

A pesquisa foi realizada no Hospital São Camilo - Cura d'Ars, localizado na Rua Costa Barros, 833 - Centro, Fortaleza - CE, 60160-280. No período entre janeiro de 2016 e outubro de 2021.

\subsection{População do estudo}

Pacientes do Hospital São Camilo - Cura d'Ars (HSC) que, durante o período acima, realizaram antibioticoterapia de alto custo e amplo espectro definidos pelo SCIH e coletaram amostras de culturas (hemocultura, urinocultura, culturas de secreções respiratórias ou outras).

\subsection{Coleta de Dados}

Foram coletados os dados clínicos, laboratoriais e prescrições de antimicrobianos dos pacientes, buscando avaliar o uso racional dos antibióticos.

\subsection{Critérios de inclusão}

Pacientes do HSC que, por qualquer motivo, estava presente em seu prontuário a prescrição de antibióticos e/ou resultados de culturas.

\subsection{Critérios de exclusão}

Pacientes com dados incompletos ou que impossibilitem a determinação dos objetivos.

\subsection{Amostra}

Formada pela população de pacientes atendidos no HSC (Fortaleza-CE) e que preencham os critérios de inclusão supracitados.

\subsection{Análise de dados}

Os dados nominais foram expressos em forma de gráficos.

\subsection{Aspectos éticos}

Esta pesquisa seguiu a Resolução 466/12 do Conselho Nacional de Saúde, que regulamenta pesquisas envolvendo seres humanos. O estudo foi aprovado pelo Comitê de Ética do Centro Universitário Christus/Unichristus com CAAE: 90842418.1.0000.5049 e parecer número 2.762.446. 


\section{Resultados}

Foram emitidos 2.733 pareceres para avaliação de uso de antibióticos pela SCIH em 2020, de janeiro a dezembro. Em 2021, de janeiro a agosto, foram emitidos 2.044 pareceres. Observa-se maiores picos de emissão de pareceres em 2020 entre abril e junho, e em 2021 entre fevereiro a abril, correspondendo aos picos de $1^{\mathrm{a}}$ e $2^{\mathrm{a}}$ onda no Brasil nos respectivos anos (Figura 1 e 2).

Figura 1. Número de pareceres mensais emitidos pelo Serviço de Controle de Infecção Hospitalar durante o ano de 2020.

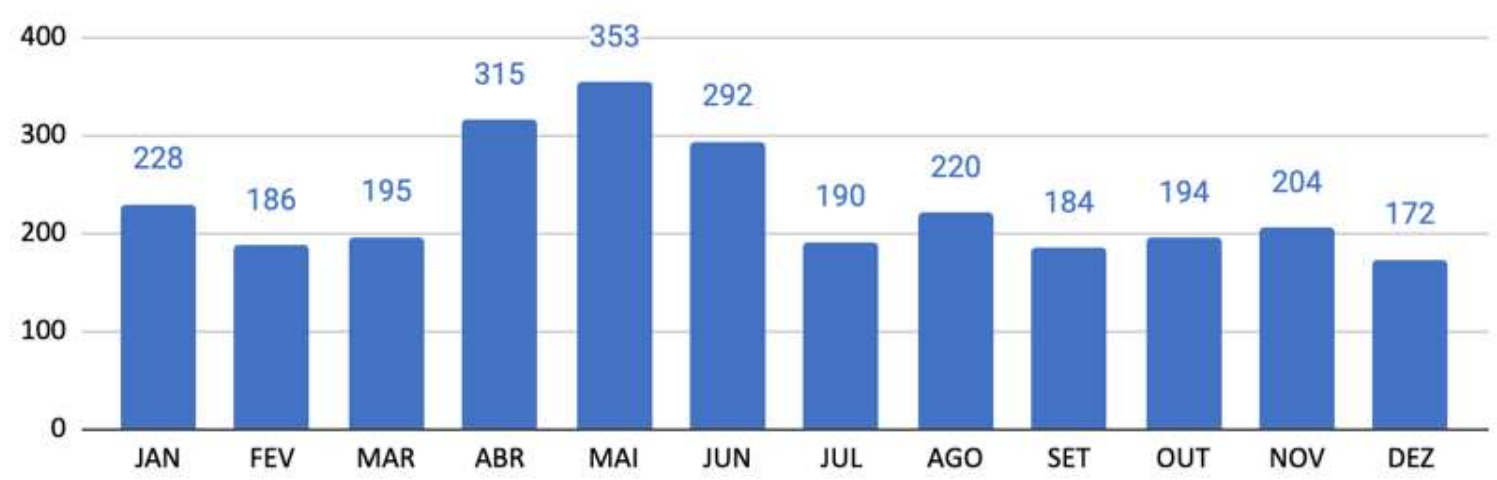

Os dados apresentados se referem ao número de avaliações de prescrição dos antimicrobianos de alto custo pelo SCIH durante o ano de 2020. Evidenciando maior aumento no período de abril a junho, coincidindo com os picos de maiores internamentos por Covid-19 no período. Fonte: Dados dos autores, referentes ao SCIH do Hospital São Camilo Fortaleza.

Figura 2. Número de pareceres mensais emitidos pelo Serviço de Controle de Infecção Hospitalar durante o ano de 2021

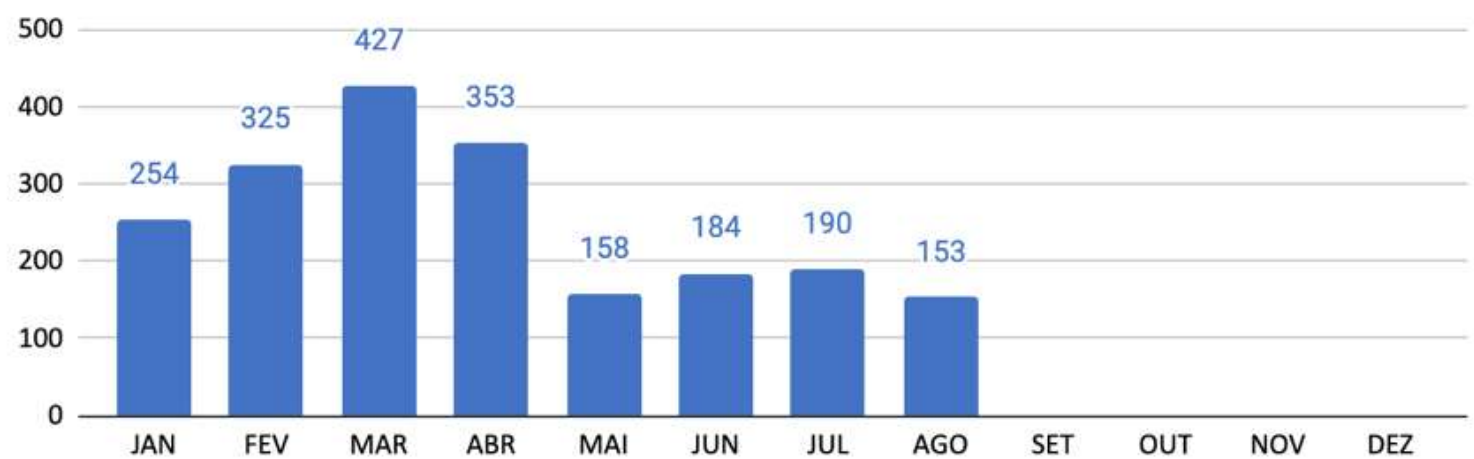

Os dados apresentados se referem ao número de avaliações de prescrição dos antimicrobianos de alto custo pelo SCIH durante o ano de 2021. Evidenciando maior aumento no período de fevereiro a abril, coincidindo com os picos de maiores internamentos por Covid-19 de paciente críticos crônicos no período avaliado de janeiro a agosto de 2021.

Fonte: Dados dos autores, referentes ao SCIH do Hospital São Camilo Fortaleza.

Avaliando o consumo de antimicrobianos de uso restrito e liberados com auditoria da SCIH, percebe-se que os antimicrobianos mais utilizados são para Gram negativos, sendo estes a Piperacilina/tazobactam e o Meropenem, tanto em 2020 quanto em 2021 (Figuras 3 e 4). 
Figura 3. Antimicrobianos de Controle Restrito pela SCIH no ano de 2020

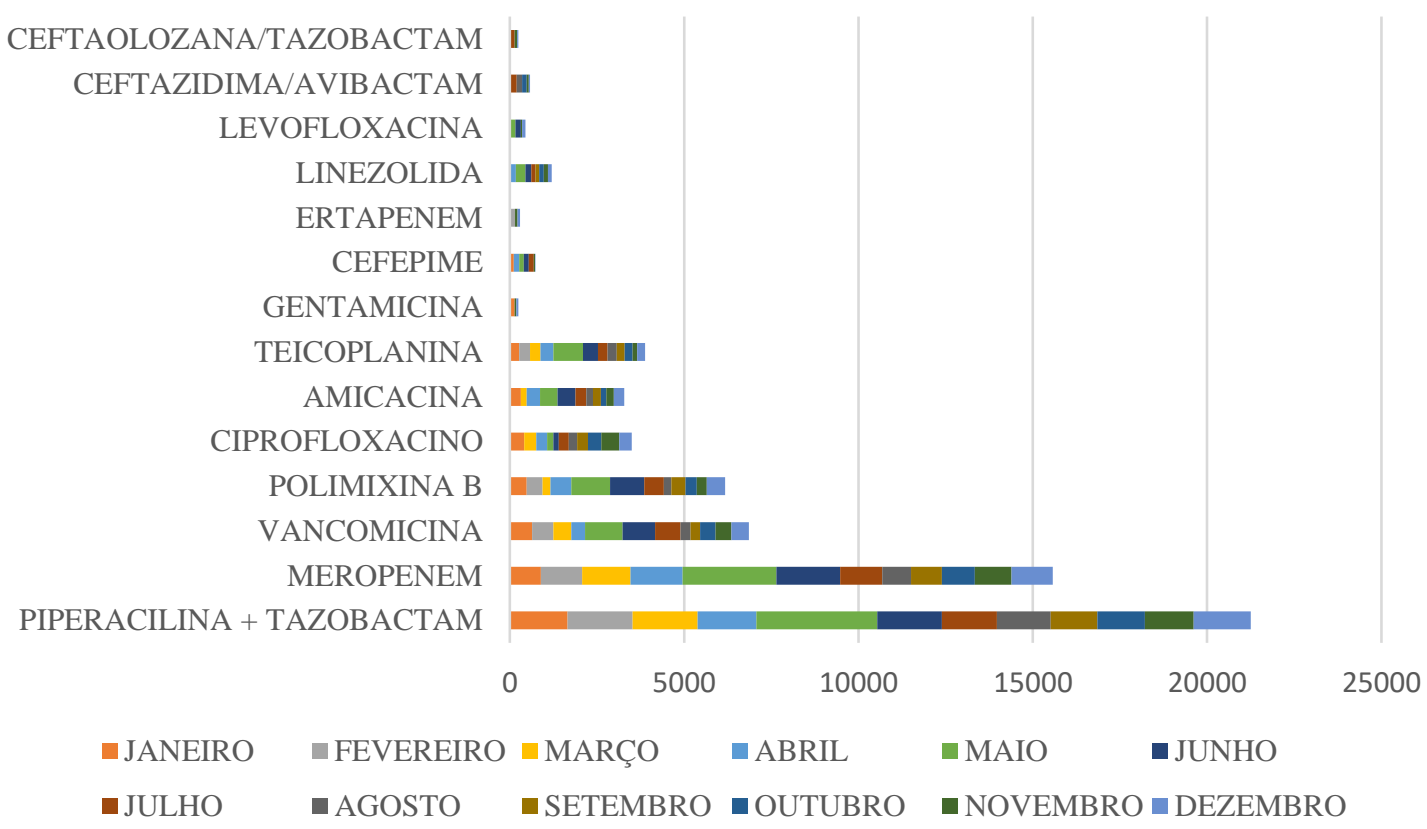

O gráfico apresenta o consumo mensal de antimicrobianos de alto custo e amplo espectro que são controlados pelo programa de uso racional de antimicrobianos da SCIH. Observa-se a maior utilização de betalactâmicos e carbapenens durante todo o ano de 2020.

Fonte: Dados dos autores, referentes ao SCIH do Hospital São Camilo Fortaleza.

Durante o ano de 2020 a utilização destes foi mais frequente entre maio e junho, e em 2021 nos meses de março e abril, sendo estes referidos meses os que refletem os pacientes com internação prolongada pós-Covid-19 e múltiplas infecções bacterianas secundárias. Observa-se também nesse período maior prescrição de Polimixina B e glicopeptídeos para Gram positivos (Vancomicina e Teicoplanina). Nesses dois anos houve um aumento na utilização de cefalosporina de espectro avançado (Ceftazidima/avibactam), sendo o aumento de 2021 reflexo do desabastecimento no país da Polimixina B (Figuras 3 e 4).

Nos últimos 20 anos, surtos causados por isolados de A. baumannii resistentes a carbapenem se tornaram uma grande preocupação mundial, e as infecções causadas por esse patógeno estão associadas ao aumento da morbidade e mortalidade. De acordo com o programa de vigilância antimicrobiana SENTRY para a América Latina, as taxas de resistência aos carbapenêmicos aumentaram quase $60 \%$ em apenas uma década entre os isolados brasileiros de A. baumannii, de 12,6\% entre 1997 e 1999 para 71,4\% entre 2008 e 2010. O grupo de Acinetobacter spp., demonstraram nos últimos anos um desaparecimento como causa importante de infecções nosocomiais na instituição do estudo atual, a despeito da grande dificuldade de antimicrobianos com cobertura adequada para seu tratamento. Porém, durante o ano de 2021, vários pacientes apresentaram infecção por esse patógeno, principalmente com uma elevada resistência a Meropenem (94,7\%) e inclusive a Polimixina B (11,1\%), (Figura 5). 
Figura 4. Antimicrobianos de Controle Restrito pela SCIH no ano de 2021

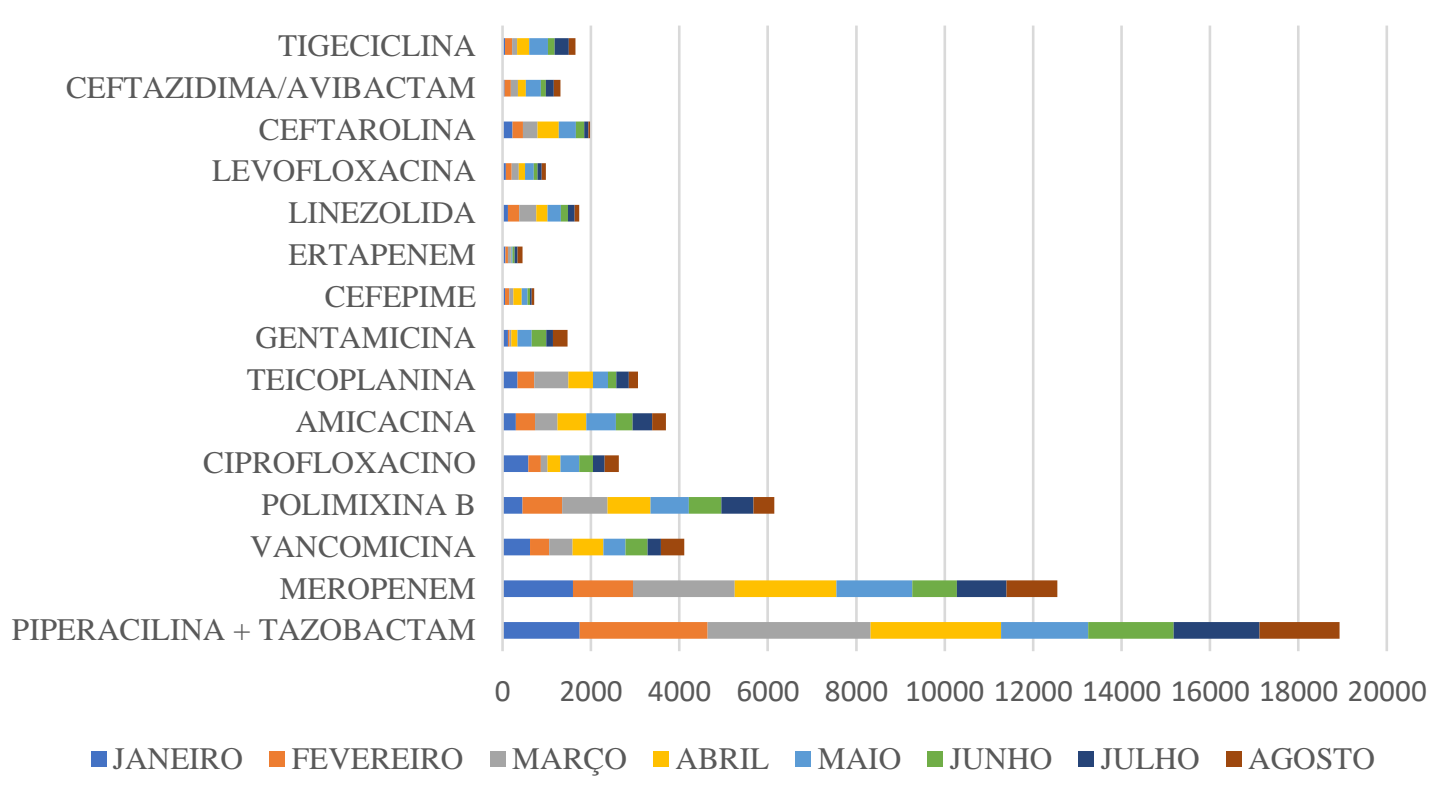

O gráfico apresenta o consumo mensal de antimicrobianos de alto custo e amplo espectro que são controlados pelo programa de uso racional de antimicrobianos da SCIH. Observa-se a maior utilização de betalactâmicos e carbapenens durante o período de janeiro a agosto de 2021. Fonte: Dados dos autores, referentes ao SCIH do Hospital São Camilo Fortaleza.

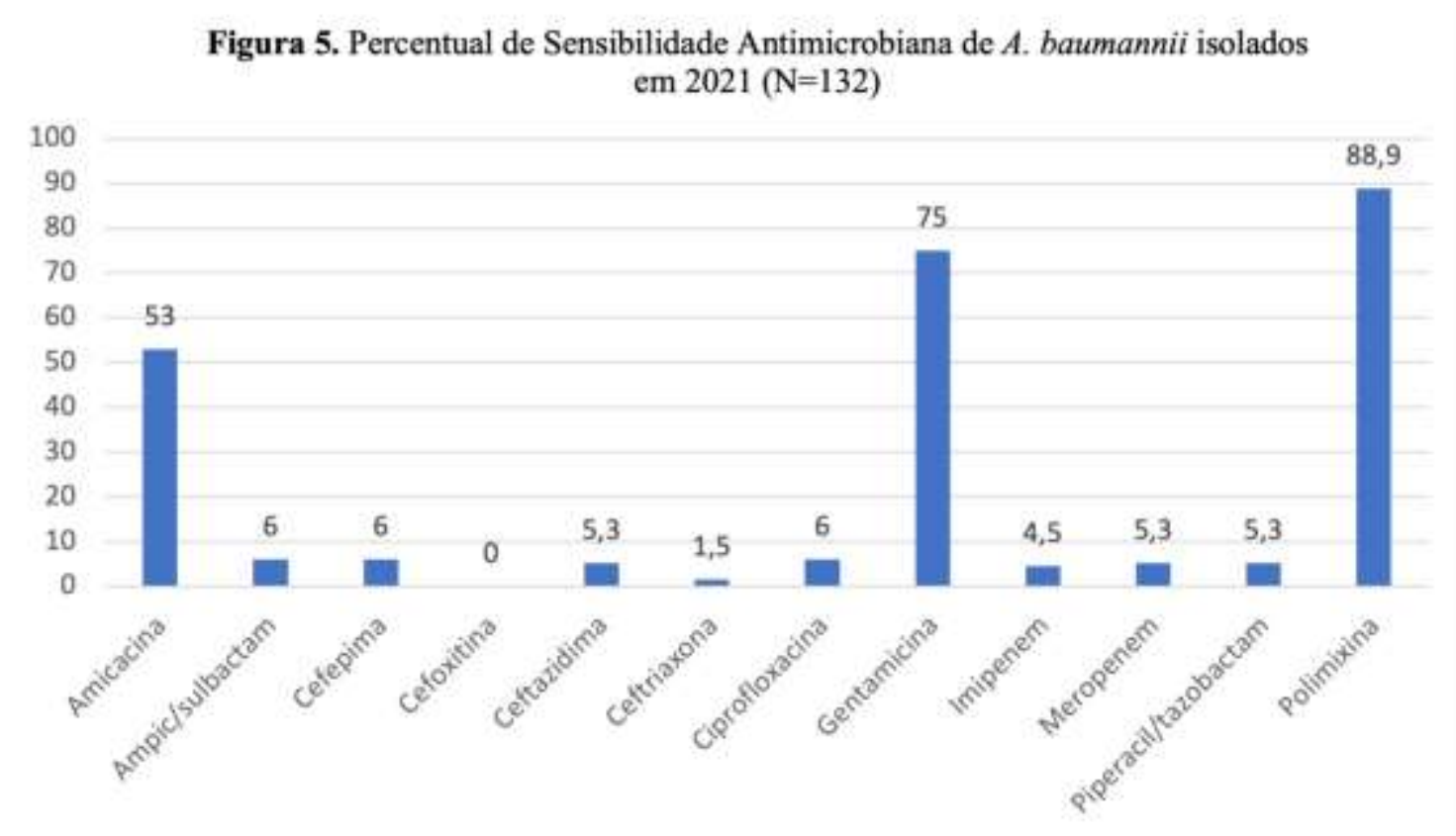

$\mathrm{N}=132$, porém Polimixina B foi realizada por microdiluicão e o $\mathrm{N}=36$.

O gráfico apresenta o perfil de sensibilidade antimicrobiana para Acinetobacter baumannii das amostras isoladas de pacientes durante o ano de 2021. Observando-se maior sensibilidade para aminoglicosídeos e polimixina que foi testada através de técnica de microdiluição em calda. Fonte: Dados dos autores, referentes ao SCIH do Hospital São Camilo Fortaleza.

Avaliar a progressão do perfil de sensibilidade microbiológica de uma instituição é importante para definir o impacto do uso racional de antimicrobianos e seu impacto direto na flora microbiana deste. Durante os últimos 5 anos foram avaliados os perfis de sensibilidade dos principais agentes causadores de infecção nosocomial neste hospital, evidenciando diferenças no curso da resistência ao longo dos anos. Ao observar a curva de sensibilidade para S. aureus percebe-se uma estabilidade quanto 
aos glicopeptídeos, embora tenha sido evidenciado previamente a ampla utilização destes antimicrobianos na instituição. Além de redução na sensibilidade a Oxacilina (Figura 6). Na avaliação de infecções por E. coli, detecta-se um perfil de sensibilidade mantido para Carbapenens, inclusive Ertapenem, assim como Aminoglicosídeos, Cefepime e Piperacilina/tazobactam (Figura 7). Portanto, para esses dois patógenos a pressão seletiva não parece ter tido impacto importante, não modificando nas escolhas de terapia empírica.

Figura 6. Avaliação do Perfil de Sensibilidade Antimicrobiana para S. aureus durante o período de 2016 a 2021 em Hospital de Fortaleza/CE.

Perfil de Sensibilidade de S. aureus

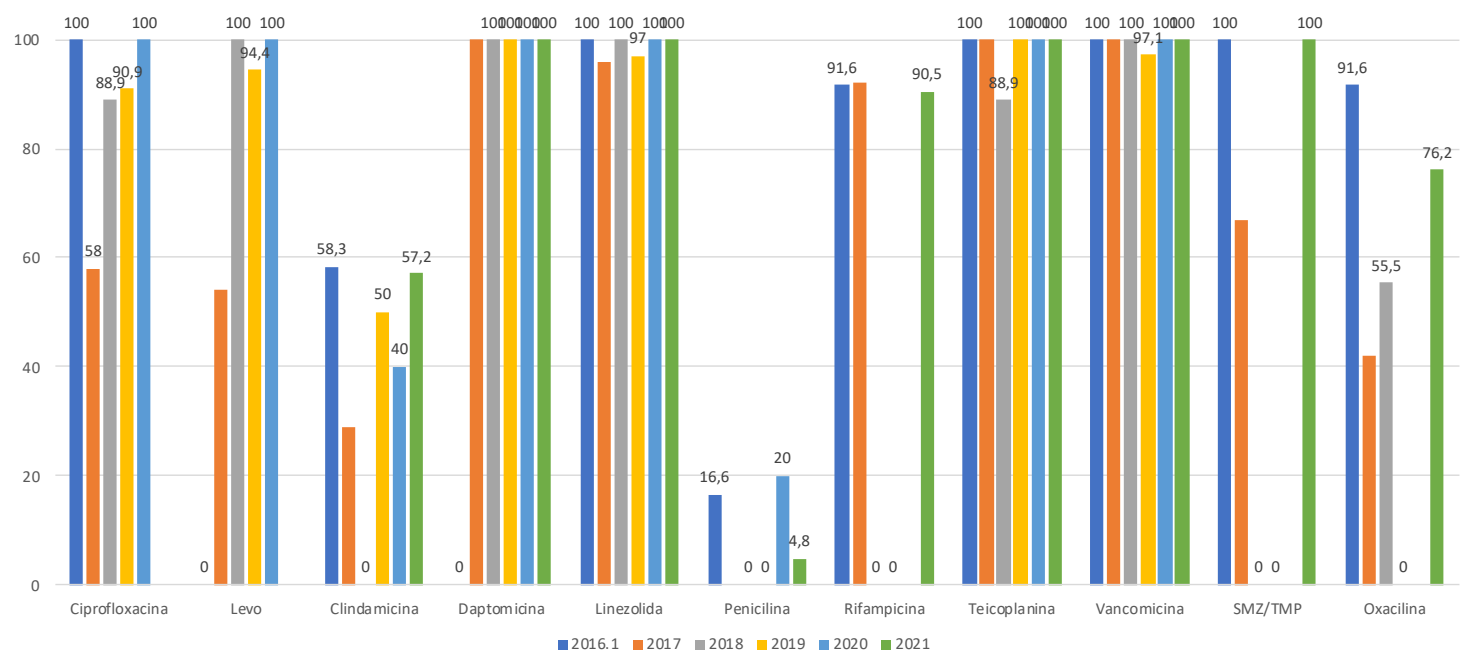

Os dados apresentados se referem ao perfil de sensibilidade antimicrobiana do S. aureus entre o período de 2016 a 2021 (5 anos de evolução), quando se avalia as mudanças no período de 2020-2021 relacionados a pandemia pela Covid-19.

Fonte: Dados dos autores, referentes ao SCIH do Hospital São Camilo Fortaleza. 
Figura 7. Avaliação do Perfil de Sensibilidade Antimicrobiana para E. coli durante o período de 2016 a 2021 em Hospital de Fortaleza/CE.

Perfil de Sensibilidade E. coli

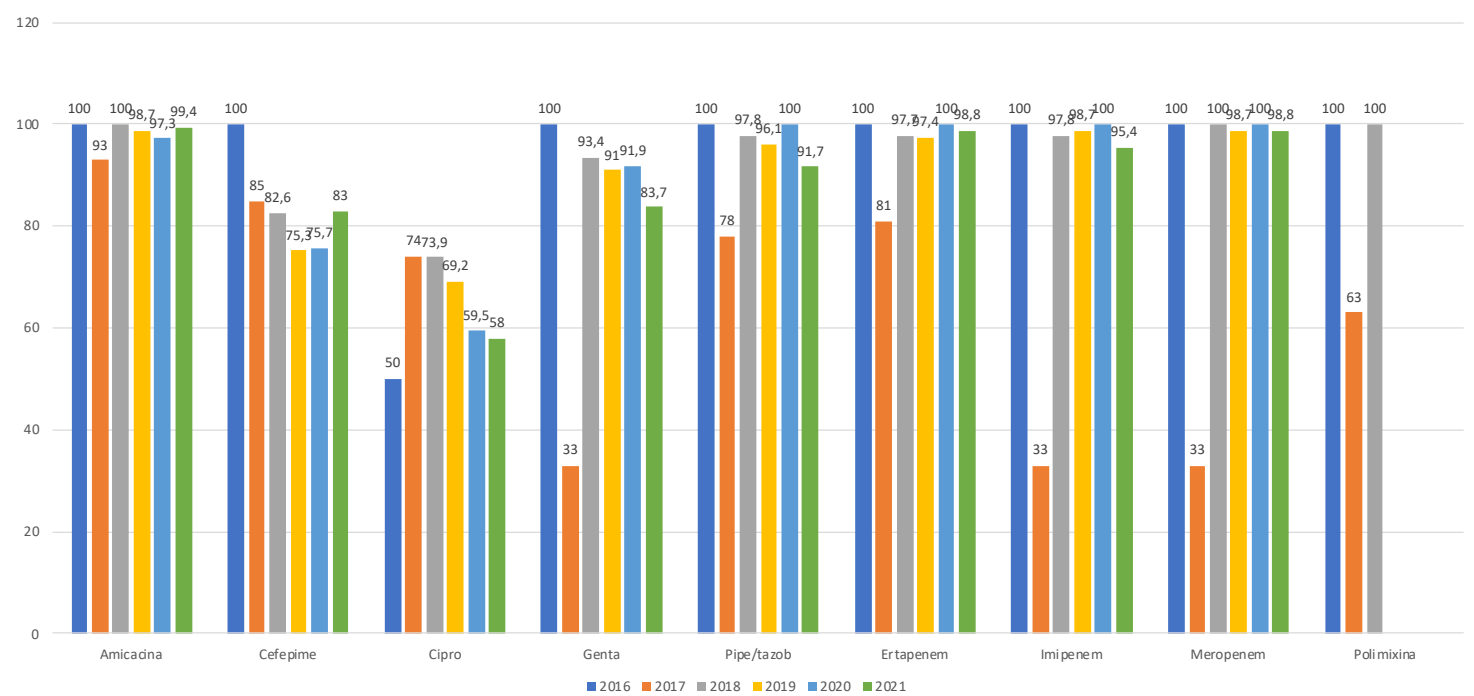

Os dados apresentados se referem ao perfil de sensibilidade antimicrobiana do E. coli entre o período de 2016 a 2021 (5 anos de evolução), quando se avalia as mudanças no período de 2020-2021 relacionados a pandemia pela Covid-19.

Fonte: Dados dos autores, referentes ao SCIH do Hospital São Camilo Fortaleza.

Ao avaliar o perfil de sensibilidade dos Gram negativos $P$. aeruginosa e K. pneumoniae, observamos um padrão no perfil de sensibilidade bastante diferente dos anteriores. Para $P$. aeruginosa observa-se uma redução drástica no último ano da sensibilidade a Piperacilina/tazobactam e Carbapenens, bem como a Polimixina B (91,7\% de sensibilidade), (Figura 8). Para K. pneumoniae os resultados foram ainda piores, evidenciando alta resistência aos Carbapenens e Polimixina B, cuja sensibilidade do último foi de apenas 76,2\%, dificultando a escolha de opções terapêuticas (Figura 9).

No cenário de alta resistência aos Carbapenens, a utilização de Polimixina B e Ceftazidima/avibactam se tornaram mais frequentes e importantes como terapia empiríca. Porém, como esperado a pressão seletiva de sua utilização também impactou diretamente no perfil de sensibilidade a estes antimicrobianos. Detectou-se redução da sensibilidade a Cefatzidima/avibactam para $P$. aeruginosa, K. pneumoniae e Serratia sp. durante o período de utilização deste antimicrobiano desde seu lançamento no mercado (Figura 10). 
Figura 8. Avaliação do Perfil de Sensibilidade Antimicrobiana para P. aeruginosa durante o período de 2016 a 2021 em Hospital de Fortaleza/CE.

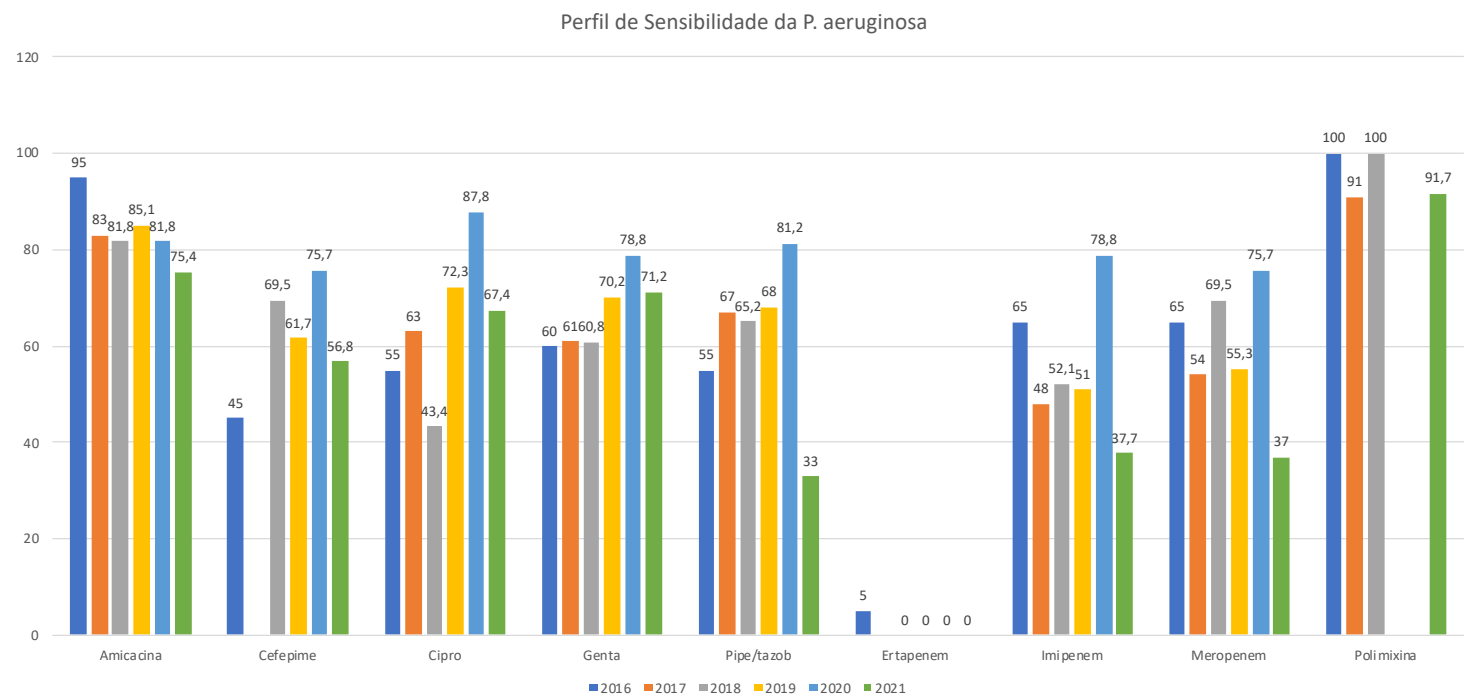

Os dados apresentados se referem ao perfil de sensibilidade antimicrobiana do P. aeruginosa entre o período de 2016 a 2021 (5 anos de evolução), quando se avalia as mudanças no período de 2020-2021 relacionados a pandemia pela Covid-19.

Fonte: Dados dos autores, referentes ao SCIH do Hospital São Camilo Fortaleza.

Figura 9. Avaliação do Perfil de Sensibilidade Antimicrobiana para K. pneumoniae durante o período de 2016 a 2021 em Hospital de Fortaleza/CE.

Perfil de Sensibilidade da K. pneumoniae

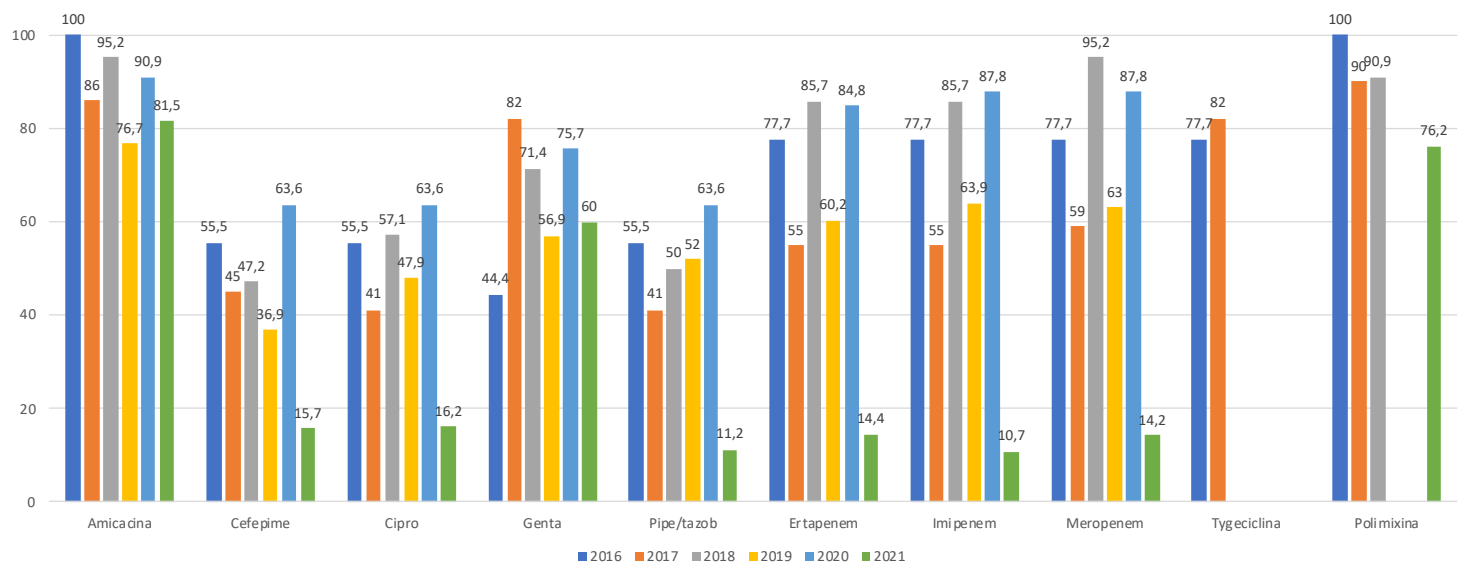

Os dados apresentados se referem ao perfil de sensibilidade antimicrobiana do K. pneumoniae entre o período de 2016 a 2021 (5 anos de evolução), quando se avalia as mudanças no período de 2020-2021 relacionados a pandemia pela Covid-19.

Fonte: Dados dos autores, referentes ao SCIH do Hospital São Camilo Fortaleza. 
Figura 10. Percentual de Sensibilidade a Ceftazidima/avibactam para Gram negativos resistentes a Carbapenem

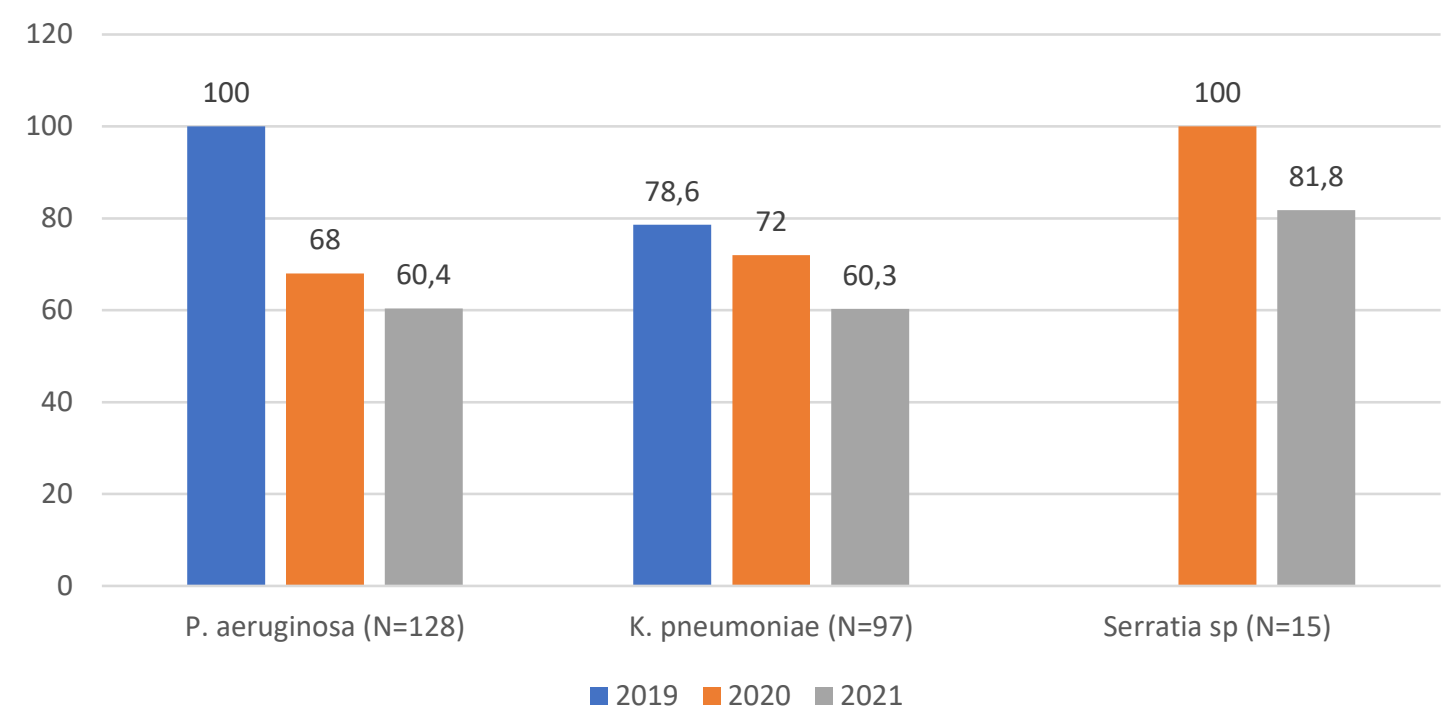

Os dados apresentados se referem ao perfil de sensibilidade a ceftazidima/avibactam, considerada cefalosporina de espectro avançado, para os principais Gram negativos causadores de infecção nosocomial: P. aeruginosa, K. pneumoniae e Serratia sp.

Fonte: Dados dos autores, referentes ao SCIH do Hospital São Camilo Fortaleza.

\section{Discussão}

Entre os estudos prévios, bacilos Gram-negativos e espécies do gênero Staphylococcus spp. são os microrganismos predominantemente identificados como a causa de infecção nosocomial nos hospitais brasileiros, corroborando os achados deste estudo (Oliveira et al., 2010. A prevalência de Gram-negativos nas instituições da América do Sul é bem conhecida, e nosso estudo evidenciou esses patógenos como mais prevalentes e o impacto da pressão seletiva ao longo do tempo (Andrade et al, 2021). Um estudo anterior observou a correlação entre a prescrição de antimicrobianos e o índice de resistência bacteriana revelando uma relação não uniforme para cada bactéria, cada agente antimicrobiano e em cada setor. Detectou aumento de isolados resistentes de $P$. aeruginosa às quinolonas, equivalene a aumento no consumo dessa classe na UTI geral, e os índices de resistência de $P$. mirabilis associados a um aumento no consumo de aminoglicosídeos e cefalosporinas (Sanches et al, 2020). No estudo de Lee et al. (2010) o aumento do uso de Levofloxacina e Ciprofloxacina, mesmo com o baixo impacto observado especificamente na resistência desta última, foi positivamente correlacionado com o aumento da resistência de P. aeruginosa às fluoroquinolonas e sua suscetibilidade foi restabelecido pela redução do uso de Levofloxacina (Lee, et al., 2010). Em nosso estudo o impacto do uso de antimicrobianos foi ainda maior, extenso aos Carbapenens e Piperacilina/tazobactam.

Em outro estudo sobre as IRAS em unidades de terapia intensiva no Brasil, demonstrou-se que os microrganismos mais prevalentes eram Staphylococcus coagulase-negativa, Pseudomonas aeruginosa e Staphylococcus aureus (Gomes et al., 2020). Observou-se em revisão integrativa que os antibióticos de maior resistência bacteriana são Piperacilina/tazobactam, Ceftriaxona, Ceftazidima, Cefotaxima, Cefepime, Imipenem e Meropenem para Escherichia coli e Klebsiella pneumoniae; Ampicilina e Vancomicina para Enterococcus faecium; Oxacilina para Staphylococcus aureus; Ciprofloxacina, Ceftriaxona e Imipenem para Acinetobacter baumannii e Pseudomonas aeruginosa; Ampicilina, Ceftriaxona e Ciprofloxacina para Enterobacteriaceae em UTIs e em outras áreas de hospitalização (Rodrigues et al., 2018). Frequentemente um grupo de bactérias está relacionado a elevados índices de resistência hospitalar: ESKAPE (Enterococcus faecium, Staphylococcus aureus, Klebsiella pneumoniae, Acinetobacter baumannii, Pseudomonas aeruginosa, Enterobacter spp.), (Zhu et al., 2014). Neste estudo, também foram encontrados altos índices de resistências pelas bactérias S. aureus, K. pneumoniae e A. baumannii. 
Durante a pandemia por Covid-19, a utilização empírica de vários desses antimicrobianos, mesmo sem indicação por tratar-se de infecção viral, podem ter impactado diretamente no aumento da resistência bacteriana em nossa instituição. Seja por incerteza no diagnóstico de infecção viral ou pela ausência de tratamento eficaz e necessidade de prescrever algum medicamento terapêutico para os pacientes, observou-se o aumento das prescrições empíricas de antibióticos durante a pandemia.

Tem-se observado um crescente aumento da resistência bacteriana aos antimicrobianos, gerando dificuldades no controle de infecções e aumento nos custos em saúde. Esses patógenos resistentes causam cerca de 700.000 mortes por ano em todo o mundo (World Health Organization, 2019).

Em estudo realizado em Cuiabá, Mato Grosso, todas as bactérias gram negativas apresentaram sensibilidade à Amicacina, exceto A. baumannii que apresentou resistência a todos os antimicrobianos testados, incluindo Amicacina, Cefepime, Ceftazidina, Ciprofloxacino, Gentamicina, Imipinem, Levofloxacino, Piperacilina/tazobactam, Sulfametoxazol/tripetoprima e sensibilidade intermediária a Ampicilina/sulbactam. Ainda em relação a A. baumannii, foram encontradas resistência a Cefepime (81\%), Ceftazidima (83,7\%), Ceftriaxona (82,6\%), Amicacina (67,4\%), Imipenem (72,3\%) e para Meropenem (75,5\%), (Ricas et al., 2013). No presente estudo, as taxas de resistência para A. baumannii foram ainda maiores, podendo ter sofrido o impacto direto do uso de múltiplos antimicrobianos consecutivos durante a pandemia. O A. baumannii tem se destacado quanto a sua alta capacidade em adquirir mecanismos de resistência às diferentes classes de antimicrobianos, além de se adaptar a condições adversas e sobreviver com maior facilidade (Bastos et al., 2020). Dessa forma, o conjunto desses fatores se traduzem em elevadas taxas de morbimortalidade, sobretudo em pacientes críticos, tornando cada vez mais restrita as opções terapêuticas. A resistência aos carbapenens pode ser explicada pela diminuição da permeabilidade das membranas externas, alteração na afinidade das proteínas ligadoras de penicilinas (PBPs) e, também, pela hiperexpressão de bombas de efluxo (Gusatti etal., 2009; Ribeiro et al., 2019). Não conseguimos identificar o mecanismo molecular de resistência no presente estudo, mas o aumento da resistência a Ceftazidima/avibactam ao longo dos anos pode indicar indiretamente o surgimento de metalo-betalactamases.

A resistência por parte da $E$. coli aos betalactâmicos está relacionada essencialmente a produção de betalactamases de espectro estendido (ESBLs) do tipo TEM ou SHV, codificadas por plasmídeos. Estas enzimas possuem a capacidade de hidrolisar penicilinas e cefalosporinas, com maior atividade hidrolítica para a Ceftazidima (entre as cefalosporinas de largo espectro) e possuem a capacidade de ser inibidas pelo ácido clavulânico (Bastos et al., 2020). Além disso, foi descoberto um novo grupo de enzimas, o CTX-M, betalactamases de espectro estendido (Rivera-Jacinto et al., 2015), que tem se tornado cada vez mais frequente em ambientes hospitalares.

Pesquisas demonstram que em junho de 2020, houve um grande aumento de Pseudomonas aeruginosa, sendo a maioria isoladas de hemoculturas ou secreções traqueais de pacientes em unidades de terapia intensiva (Zhang Et. al., 2020). Assim, como foi evidenciado no presente estudo, o aumento no isolamento e prevalência dessa bactéria e uso com maior frequência de Carbapenens durante a pandemia podem ter tido impacto direto no aumento da resistência a este patógeno.

Existe ainda um problema maior, como a resistência dos não fermentadores (Pseudomonas aeruginosa, Acinetobacter baumannii, Stenotrophomonas maltophilia e Burkholderia cepacia), eles possuem extrema resistência intrínseca e ainda são associados a resistências adquiridas no âmbito hospitalar, através da medicação. Já em casos de bactérias gram-positivas (Staphylococcus sp., Enterococcus sp., Streptococcus sp.) ou enterobactérias (Escherichia coli, Proteus sp., Providencia sp., Serratia sp., Klebsiella, Enterobacter sp.), podemos observar resistência a Meticilina, Vancomicina e Teicoplanina, no caso de bactérias gram-positivas e das enterobactérias as bactérias produtoras de betalactamase, o que significa resistência as cefalosporinas e também as produtoras de KPC (Lansbury et. al., 2020).

De acordo com trabalhos realizados na cidade de Wuhan de $71 \%$ a $95 \%$ das bactérias de pacientes com o novo vírus tratado com antibióticos, possuem grande resistência, complicando assim o seu tratamento e levando a um aumento das 
superbactérias e da taxa de mortalidade no país. Os antibióticos utilizados de forma preventiva ampliam a resistência bacteriana levando assim a maior mortalidade (Hsu, 2020).

A escassa informação ou produções de informações falsas durante a pandemia está levando a um aumento na automedicação ou terapias enganosas para a Covid-19, principalmente na Ásia, onde acima de 70\% dos pacientes utilizam antibióticos, mesmo apenas $10 \%$ realmente apresentarem infecções bacterianas ou fúngicas. Porém, a utilização desses antibióticos e principalmente os de amplo espectro para tratar o novo vírus, elimina diversas bactérias colonizantes da microbiota natural, estimulando o aumento da resistência antimicrobiana (Rawson, et al., 2020).

De acordo com trabalhos publicados observa-se que a cada sete pacientes um adquiriu infecção secundária por bactérias, desses pacientes, $50 \%$ morreram por conta dessas co-infecções, tornando-se um grande desafio para o uso racional de antimicrobianos, piorando e aumentando a quantidade de mortes em todo o sistema de saúde e levando a mais casos de infecção nosocomial (Zhou et al. 2020; Silva et al, 2021). Percebe-se, portanto, a dificuldade na tomada de decisão por serviços de controle de infecção hospitalar e equipe prescritora, o dilema entre evitar o uso demasiado de antimicrobianos levando a resistência e a hesitação na prescrição destes com desfecho desfavorável para o paciente. Devendo-se prevalecer o bom senso nas decisões e avaliações diárias sobre necessidade de utilização de antimicrobiano pelo paciente, tempo de suspensão ou troca sempre que possível com auxílio de exames de cultura.

\section{Considerações Finais}

O estudo evidenciou aumento da resistência antimicrobiana pelos principais patógenos Gram negativos responsáveis por infecções nosocomiais, como $P$. aeruginosa e $K$. pneumoniae, inclusive para novas cefalosporinas como Ceftazidima/avibactam e Polimixinas, que podem ter impacto nas opções terapêuticas e mortalidade do paciente. A utilização maior empírica de Piperacilina/tazobactam e Carbapenens no período de pandemia pela Covid-19 pode ter sido responsável pela maior pressão seletiva e resistência bacteriana detectadas no último ano, bem como o ressurgimento do A. baumannii com alto perfil de resistência. O uso racional de antimicrobianos é uma medida essencial para controle do aumento progressivo de resistência aos antimicrobianos, devendo ser continuamente implementada pelo risco de falta de opções em um futuro próximo.

A continuidade do trabalho atual deve ser mantida na perspectiva de melhor monitoramento do perfil microbiológico da instituição que guia as terapias empíricas e os protocolos para utilização racional dos antimicrobianos. Sugerimos que medidas de maior controle de antibióticos sejam instituídas nos nosocômios, e que as instituições façam registros precisos dos impactos no perfil dos patógenos que causam infecções dentro dos serviços de institucionalização hospitalar para que o Brasil tenha um monitoramento mais eficaz das suas ações de prevenção e controle.

\section{Agradecimentos}

Agradecemos a todo o Serviço de Controle de Infecção Hospitalar (SCIH) do Hospital São Camilo de Fortaleza, principalmente Matheus César Dias Patrício, Francelene da Silva Almeida, Patrícia Borges Barjud Coelho e Virna Yane Bezerra Lopes, com os quais seria impossível enfrentar uma pandemia de tamanha magnitude. Time cuja dedicação e competência não apresentam precedentes.

\section{Referências}

Adebisi, Y. A., Alaran, A. J., Okereke, M., Oke, G. I., Amos, O. A., Olaoye, O. C., Oladunjoye, I., Olanrewaju, A. Y., Ukor, N. A., \& Lucero-Prisno, D. E. (2021). Covid-19 and Antimicrobial Resistance: A Review. Infect Dis (Auckl). 14:11786337211033870.

Andrade, C. W. Q., Silva, K. S. B., Santana, M. M. R., Oliveira, A. V., Guimarães, M. D., \& Naue, C. R. (2021). Etiologia e resistência de isolados bacterianos de hemoculturas da Sala de Cuidados Intermediários de um Hospital Universitário em Pernambuco. Research, Society and Development, 10 (7), e37510716605. 
Aquino, D. S. (2008). Por que o uso racional de medicamentos deve ser uma prioridade? Ciênc. saúde coletiva, 13, $733-736$.

Bastos, I. D. M., Bastos, B. D. M., Silva, C. F., Silva, K. S. B., \& Naue, C. R. (2020). Perfil bacteriano de amostras microbiológicas de pacientes internados na Clínica Cirúrgica de um Hospital Universitário de Pernambuco. VITTALLE-Revista de Ciências da Saúde, 32(1), $108-121$.

Dandolini, B. W., Batista, L. B., Souza, L. H. F., Galato, D., \& Piovezan, A. P. (2012). Uso racional de antibióticos: uma experiência para educação em saúde com escolares. Ciênc. saúde coletiva, 17(5), 1323-1331.

de Kraker, M. E., Stewardson, A. J., \& Harbarth, S. (2016). Will 10 million People Die a Year due to Antimicrobial Resistance by 2050? PLoS medicine, 13(11), e1002184.

FDA, CDRH, CBER. (2017). Use of real-world evidence to support regulatory decision-making for medical devices. Guidance for industry and food and drug administration staff preface public comment.

Fiol, F. S. D., Barberato-Filho, S., Lopes, L. C., \& Toledo, M. I. (2010) Level of patient information on antibiotic use. Braz. J. Pharm. Sci., São Paulo, v. 46, n. 3, p. $437-444$

Gomes, A. A. G., Silva, M. R. da Garcês, T. C. de C. S., Pinto, A. S. B., Andrade, S. M. O. de, Saraiva, E. R., Brito, S. M. S., Gadelha, D. dos S. G., \& Andrade, A. R. O. de. (2020). Infecções relacionadas à assistência em saúde em unidades de terapia intensiva no Brasil. Revista Eletrônica Acervo Saúde, 12(11), e4665-e4665.

Gusatti, C. de S., Ferreira, A. E., Fuentefria, D. B., \& Corção, G. (2009). Resistência a $\beta$-lactâmicos em Acinetobacter spp isolados de efluente hospitalar no sul do Brasil. Revista da Sociedade Brasileira de Medicina Tropical, 42(2), 183-187

Hawkey, P. M. (2015). Multidrug-resistant Gram-negative bacteria: a product of globalization. Journal of Hospital Infection, 89(4).

Helke, K. L., McCrackin, M. A., Galloway, A. M., Poole, A. Z., Salgado, C. D., \& Marriot, B. P. (2016). Effects of antimicrobial use in agricultural animals on drug-resistant foodborne salmonellosis in humans: A systematic literature review. Critical Reviews in Food Science and Nutrition, 57(3).

Hsu, J. (2020). How Covid-19 is accelerating the threat of antimicrobial resistance. BMJ, 369.

Kalpoe, S., Sonnenberg, E., Factor, S. H., Martin, J. R., Patel, T. S. G., \& Huprikar, S. (2012). Mortality associated with carbapenem-resistant Klebsiella pneumoniae infections in liver transplant recipients. Liver Transplantation, 18(4).

Lansbury, L., Lim, B., Baskaran, V., \& Lim, W. S. (2020). Co-infections in people with Covid-19: a systematic review and meta-analysis. Journal of Infection.

Lee, Y. J., Liu, H. Y., Lin, Y. C., Sun, K. L., Chun, C. L., \& Hsuehe, P. R. (2010). Fluoroquinolone resistance of Pseudomonas aeruginosa isolates causing nosocomial infection is correlated with levofloxacin but not ciprofloxacin use. International Journal of Antimicrobiol Agents, 35(3).

Luepke, K. H., Suda, K. J., Boucher, S. H., Russo, R. L., Bonney, M. W., Hunt, T. D., \& Mohr III, J. F. (2013). Past, present, and future of antibacterial economics: increasing bacterial resistance, limited antibiotic pipeline, and social implications. Pharmacotherapy, 37(1).

Marra, A. R., Camargo, L. F. A., Pignatari, A. C. C., Sukiennik, T., Behar, P. R. P., Medeiros, E. A. S., Ribeiro, J., Girão, E., Correa, L., Guerra, C., Brites, C., Pereira, A. P., Carneiro, I., Reis, M., Souza, Tranchesi, R., Barata, C. U., Edmond, M. B. and Brazilian SCOPE Study group. (2011). Nosocomial bloodstream infections in Brazilian hospitals: analysis of 2,563 cases from a prospective nationwide surveillance study. Clin Microbiol. May;49(5):1866-71.

Mota, D. M., Silva, M. G. C., Sudo, E. C., \& Ortun, V. (2008). Uso racional de medicamentos: uma abordagem econômica para tomada de decisões. Ciênc. saúde coletiva, 13, 589-601.

Nieuwlaat, R., Mbuagbaw, L., Mertz, D., Burrows, L. L., Bowdish, D. M. E., Moja, L., Wright, G. D., \& Schünemann, H. J. (2021). Coronavirus Disease 2019 and Antimicrobial Resistance: Parallel and Interacting Health Emergencies. Clin Infect Dis. 72(9):1657-1659.

Oliveira, A. C., Kovner, C. T., \& da Silva, R. S. (2010) Nosocomial infection in an intensive care unit in a Brazilian university hospital. Revista LatinoAmericana de Enfermagem, 18(2).

O'Neill J. Review on Antimicrobial Resistance Antimicrobial Resistance: Tackling a crisis for the health and wealth of nations. London: Review on Antimicrobial Resistance; 2014

Opatowski, M., Brun-Buisson, C., Touat, M., Salomon, J., Guillemot, D., Tuppin, P., \& Watier, L. (2021). Antibiotic prescriptions and risk factors for antimicrobial resistance in patients hospitalized with urinary tract infection: a matched case-control study using the French health insurance database (SNDS). BMC Infect Dis 21, 571

Pinto, M. C. X., Ferre, F., \& Pinheiro, M. L. P. (2012). Potentially inappropriate medication use in a city of Southeast Brazil. Braz. J. Pharm. Sci., 48(1), 79-86.

Rauber, C., Feltrin, M. R., \& Piovezan, A. P. (2009). Evaluation of antibiotics dispensing profile in Tubarão, Santa Catarina, Brazil. Braz. J. Pharm. Sci., 45(4), 787-793.

Rawson, T. M., Moore, L. S., Zhu, N., Ranganathan, N., Skolimowska, K., Gilchrist, M. \& Holmes, A. (2020). Bacterial and fungal co-infection in individuals with coronavirus: A rapid review to support Covid-19 antimicrobial prescribing. Clinical Infectious Diseases.

Rivera-Jacinto, M., Rodríguez-Ulloa, C., Flores Clavo, R., Serquén López, L., \& Arce Gil, Z. (2015). Betalactamasas de espectro extendido tipo TEM y CTXM en Klebsiella spp y Escherichia coli aisladas de superficies de ambientes hospitalarios. Revista Peruana de Medicina Experimental y Salud Pública, 32, 752755 .

Ricas, R. V., Marques, T. C., \& Yamamoto, A. C. A. (2013). Perfil de resistência de Acinetobacter baumannii a antimicrobianos em um hospital universitário de Cuiabá-MT. Infarma Ciências Farmacêuticas, 25(4), 178-181. 
Research, Society and Development, v. 11, n. 1, e58211125382, 2022

(CC BY 4.0) | ISSN 2525-3409 | DOI: http://dx.doi.org/10.33448/rsd-v11i1.25382

Rodrigues, T. S., dos Santos, A. M. R., Lima, P. C., Moura, M. E. B., Goiano, P. D. D. O. L., \& da Silva Fontinele, D. R. (2018). Resistência bacteriana a antibióticos na Unidade de Terapia Intensiva: revisão integrativa. Revista Prevenção de Infecção e Saúde, 4.

Sanches, F. K., Volcão, L. M., von Groll, A., Silva Junior, F. M. R., Silva, P. E. A., \& Ramos, D. F. (2020). Uso de antibióticos e associação com resistência bacteriana em um hospital no Sul do Brasil. Research, Society and Development, 9(6), e154963405.

Silva, K. M. R., Oliveira, R. M. A., Araújo, A. J. R. O., Paula, C. C. C., Tavares, P. S., Santana, F. S., Marinho, A. L. S., Melo, D. T., Velôso, D. S., Vieira, J. F. P. N., Silva, A. M., Silva, H. J. N., Silva, A. E. P., Silva, M. R., Mattos, M. L. F. R. (2021). Implicações do uso de antibióticos durante a pandemia de Covid19. Research, Society and Development, 10(7), e20210715684.

Zangirolami-Raimundo, J., Echeimberg, J. de O., \& Leone, C. (2018). Research methodology topics: Cross-sectional studies. Journal of Human Growth and Development, 28(3), 356-360.

Song, J. W., \& Chung, K. C. (2010). Observational studies: cohort and case-control studies. Plastic and reconstructive surgery, 126(6), $2234-2242$.

Zhang, J., Ma, X., Yu, F., Liu, J., Zou, F., Pan, T., \& Zhang, H. (2020). Teicoplanin potently blocks the cell entry of 2019-nCoV. BioRxiv.

Zhou, F., Yu, T., Du, R., Fan, G., Liu, Y., Liu, Z. \& Guan, L. (2020). Clinical course and risk factors for mortality of adult in patients with Covid-19 in Wuhan, China: a retrospective cohort study. The Lancet, 395(10229), P1054-1062.

Zhu, H., Swierstra, J., Wu, C., Girard, G., Choi, Y. H., van Wamel, W., \& van Wezel, G. P. (2014). Eliciting antibiotics active against the ESKAPE pathogens in a collection of actinomycetes isolated from mountain soils. Microbiology, 160(8), 1714-1725.

World Health Organization. (2019) No Time to Wait: Securing the future from drug-resistant infections. http://www.who.int/antimicrobialresistance/interagency-coordination-group/final-report/en/

World Health Organization (WHO). High levels of antibiotic resistance found worldwide, new data shows. http://www.who.int/news-room/detail/29-01-2018high-levels-of-antibiotic-resistance-found-worldwide-new-data-shows. 